\title{
Nonfragile Robust Model Predictive Control for Uncertain Constrained Systems with Time-Delay Compensation
}

\author{
Wei Jiang, Hong-li Wang, Jing-hui Lu, Wei-wei Qin, and Guang-bin Cai \\ Xian Institute of High-Tech, Xian 710025, China \\ Correspondence should be addressed to Wei Jiang; yixiantian123456@126.com
}

Received 4 February 2016; Revised 6 June 2016; Accepted 13 June 2016

Academic Editor: Gabriele Cazzulani

Copyright ( 2016 Wei Jiang et al. This is an open access article distributed under the Creative Commons Attribution License, which permits unrestricted use, distribution, and reproduction in any medium, provided the original work is properly cited.

\begin{abstract}
This study investigates the problem of asymptotic stabilization for a class of discrete-time linear uncertain time-delayed systems with input constraints. Parametric uncertainty is assumed to be structured, and delay is assumed to be known. In Lyapunov stability theory framework, two synthesis schemes of designing nonfragile robust model predictive control (RMPC) with time-delay compensation are put forward, where the additive and the multiplicative gain perturbations are, respectively, considered. First, by designing appropriate Lyapunov-Krasovskii (L-K) functions, the robust performance index is defined as optimization problems that minimize upper bounds of infinite horizon cost function. Then, to guarantee closed-loop stability, the sufficient conditions for the existence of desired nonfragile RMPC are obtained in terms of linear matrix inequalities (LMIs). Finally, two numerical examples are provided to illustrate the effectiveness of the proposed approaches.
\end{abstract}

\section{Introduction}

Model predictive control (MPC), also known as receding horizon control (RHC), is an online control scheme based on the conventional optimal control that is obtained by minimization or minimaximization of some performance criteria either for a fixed finite horizon or for an infinite horizon. It is one of the few areas that have received ongoing interests from researchers in both the industrial and the academic communities. For the state of the art related to the research on MPC, recent survey papers in [1-4] can be further referred to. The success of MPC is mainly due to its ability to handle control problems for multivariable systems subject to state, input, and output constraints. In this line of research, precise plant models are quite necessary. However, the inherent time delays and parameter uncertainties contained in the dynamical behavior of many physical processes are inevitable, especially during many chemical processes where the MPC has been mainly applied. To solve these problems, increasing interests have been paid to the study of the robust MPC (RMPC) techniques for linear or nonlinear time-delay systems with the polytopic or structured type of uncertainties, for example, Kothare et al. [5], Jeong and Park [6], Lu and Shao [7], Ding et al. [8, 9], Ding et al.
[10], Chen et al. [11], and references therein. To reduce the conservativeness, Qin et al. in [12,13] have proposed several novel memory state feedback RMPC algorithms for timedelayed uncertain linear systems with input constraints by the Lyapunov method, where the gain matrixes of the memory state feedback controllers consisting of both the current state and single/multiple known/unknown time-delayed states are incorporated simultaneously. Moreover, to further reduce the conservativeness and the online computational burden caused by real-time iterative optimization procedures, several ad hoc strategies such as parameter-dependent Lyapunov functions, time-varying terminal constraint sets, polyhedral invariant sets, tube invariant sets, aggregation optimization scheme, and offline design/online synthesis have been effectively introduced to RMPC algorithms (see the research papers [14-19] and references therein).

From the literature review, it is clear that an implicit assumption in the previous controllers design is that the controller will be implemented exactly. However, in practice, the parameters of the controller are possible to accrue some gain perturbations due to finite word length, the existence of the parameter drift, the round-off errors in numerical computations, or degradations of actuator effectiveness, which has been recognized as the so-called fragility problem of 
controllers, to name a few, [20-22] and references therein. A relatively minor perturbation of the controller gains may lead to performance degradation and instability. Therefore, to design a controller that is insensitive to som e amount of errors, the research of nonfragile control is more than important and significant.

Motivated by the aforementioned discussions, this paper will focus on the problem of designing nonfragile RMPC algorithms for uncertain constrained systems with timedelay compensation. Compared with the existing researches, the main contributions of this study can be summarized in following aspects. First, the idea of nonfragile control is introduced into the RMPC problems for uncertain systems with time delays. In [5-13], RMPC problems for uncertain systems with time delays have been investigated, but the potential gain perturbation during the controller implementation stages has not been taken into consideration. Second, nonfragile MPC problems with additive and multiplicative gain perturbations are, respectively, studied. The analysis approach in [23] can only be applied to the case of additive gain perturbations and is conservative since its state feedback controller only consists of current state. Third, all the conditions are presented in terms of LMIs, which can be easily calculated through using MATLAB LMI control toolbox [24]. Finally, some numerical simulations are presented to illustrate the effectiveness and the preponderance of the proposed design methods.

The rest of this paper is organized as follows. Section 2 presents the targeted systems, assumptions, and the associated problem formulation. Section 3 supplies nonfragile robust state feedback controllers with time-delay compensation. Section 4 minimizes the upper bound of infinite horizon cost that satisfies the sufficient conditions, in terms of linear matrix inequalities (LMIs). Section 5 illustrates the performance of the proposed controllers through two numerical examples. Finally, in Section 6, some concluding remarks are given.

Notation. Throughout this paper, $\mathbf{R}^{n}$ and $\mathbf{R}^{m \times n}$ represent the $n$-dimensional Euclidean space and the set of all $m \times n$ real matrixes. $\mathbf{I}$ and $\mathbf{0}$ denote the identity matrix and zero matrix with compatible dimensions. For any matrix $\mathbf{X} \in \mathbf{R}^{m \times n}, \mathbf{X}^{T}$ and $\mathbf{X}^{-1}$ denote the transpose and the inverse of $\mathbf{X}$ (in this case, $\mathbf{X}$ is square, namely, $m=n$ ), respectively. “*” is used to represent a term that is induced by symmetry in symmetric block matrixes or complex matrix expressions and $\|\cdot\|$ refers to the Euclidean vector norm or spectral matrix norm. Matrix inequality $\mathbf{X}>0(\mathbf{X}<0)$ means that $\mathbf{X}$ is positive definite (negative definite). Matrixes are assumed to be compatible for algebraic operations if their dimensions are not explicitly stated.

\section{Preliminaries and Problem Formulation}

The following discrete-time uncertain time-delay systems are considered:

$$
\begin{aligned}
\mathbf{x}(k+1)= & (\mathbf{A}+\Delta \mathbf{A}(k)) \mathbf{x}(k)+\mathbf{B u}(k) \\
& +\left(\mathbf{A}_{d}+\Delta \mathbf{A}_{d}(k)\right) \mathbf{x}(k-d),
\end{aligned}
$$

$$
\begin{aligned}
\mathbf{x}(k) & =\boldsymbol{\vartheta}(0), \quad k \in[-d, 0], \\
\|\mathbf{u}(k)\|_{2} & \leq u_{\max }, \quad u_{\max }>0, \forall k \in[0, \infty),
\end{aligned}
$$

where $\mathbf{x}(k) \in \mathbf{R}^{n}$ is the state, $\mathbf{u}(k) \in \mathbf{R}^{m}$ is the control input, $\boldsymbol{\vartheta}(0)$ is the initial condition, $\mathbf{A}$ and $\mathbf{A}_{d}$ are known constant matrixes with appropriate dimensions, $\mathbf{B}$ denotes the input matrix, and $d$ is a time-delayed constant. The structured uncertainties $\left[\Delta \mathbf{A}(k), \Delta \mathbf{A}_{d}(k)\right]$ are time-varying matrixes satisfying the following norm-bounded conditions:

$$
\begin{aligned}
\Delta \mathbf{A}(k) & =\mathbf{E} \Delta_{1}(k) \mathbf{F}, \\
\Delta \mathbf{A}_{d}(k) & =\mathbf{H} \Delta_{2}(k) \mathbf{G},
\end{aligned}
$$

where E, F, H, G denote corresponding given scaling matrixes and $\Delta_{i}(k), i=1,2$, are unknown time-varying matrixes with Lebesgue-measurable elements satisfying

$$
\Delta_{i}^{T}(k) \Delta_{i}(k) \leq \mathbf{I}, \quad i=1,2 .
$$

The unknown matrixes $\Delta_{i}(k), i=1,2$, contain uncertain parameters in the linear part of the system and affect the elements of nominal matrixes $\mathbf{A}$ and $\mathbf{A}_{d}$. The parameter uncertainty structure in (2) has been widely used in the problem of robust stabilization of uncertain systems and can represent parameter uncertainty in many physical cases. It is assumed that systems (1) are stabilizable for the existence of a stabilizing feedback control and that state $\mathbf{x}(k)$ is available at each sampling time instant $k$. In the following part, three lemmas that are necessary to establish the main results of this paper will be presented.

Lemma 1 (see $[12,13]$ ). Given any vectors $\mathbf{Z}, \mathbf{Y} \in \mathbf{R}^{n}$ and any positive definite symmetric matrix $\mathbf{P} \in \mathbf{R}^{n \times n}$, the following inequality holds:

$$
2 \mathbf{Z}^{T} \mathbf{P Y} \leq \mathbf{Z}^{T} \mathbf{P Z}+\mathbf{Y}^{T} \mathbf{P Y}
$$

Lemma 2 (see [25]). Given matrixes A, L, E, F with appropriate dimensions and that $\mathbf{F}$ is an unknown matrix satisfying $\|\mathbf{F}\|_{2} \leq 1$, then, for any positive matrix $\mathbf{P}$ satisfying $\mathbf{P}-\varepsilon \mathbf{L} \mathbf{L}^{T}>0$ and a real constant $\varepsilon>0$, the following inequality holds:

$$
\begin{aligned}
(\mathbf{A} & +\mathbf{L F E})^{T} \mathbf{P}^{-1}(\mathbf{A}+\mathbf{L F E}) \\
& \leq \mathbf{A}^{T}\left(\mathbf{P}-\varepsilon \mathbf{L L}^{T}\right)^{-1} \mathbf{A}+\varepsilon^{-1} \mathbf{E}^{T} \mathbf{E} .
\end{aligned}
$$

Lemma 3 (see $[23,24]$ ). Given matrix $\mathbf{Q}=\mathbf{Q}^{T}, \mathbf{H}, \mathbf{E}$ with appropriate dimensions, then

$$
\mathbf{Q}+\mathbf{H F E}+(\mathbf{H F E})^{T}<0
$$

For all $\mathbf{F}$ satisfying $\|\mathbf{F}\| \leq 1$, there exists a real constant $\varepsilon>0$ such that

$$
\mathbf{Q}+\varepsilon \mathbf{H H}^{T}+\varepsilon^{-1} \mathbf{E}^{T} \mathbf{E}<0 .
$$




\section{Design of Nonfragile State Feedback RMPC with Time-Delay Compensation}

In this section, the focus will be on the design of nonfragile state feedback RMPC with time-delay compensation including different gain perturbation cases. To begin with, two nonfragile state feedback control laws $\mathbf{u}_{A}(k+i \mid k)$ and $\mathbf{u}_{M}(k+$ $i \mid k)$ with additive gain perturbation and multiplicative gain perturbation are respectively described as follows:

$$
\begin{aligned}
\mathbf{u}_{A}(k+i \mid k)= & \left(\mathbf{K}_{A}+\Delta \mathbf{K}_{A}(k)\right) \mathbf{x}(k+i \mid k) \\
& +\mathbf{K}_{A d} \mathbf{x}(k+i-d \mid k), \\
\mathbf{u}_{M}(k+i \mid k)= & \left(\mathbf{K}_{M}+\Delta \mathbf{K}_{M}(k)\right) \mathbf{x}(k+i \mid k) \\
& +\mathbf{K}_{M d} \mathbf{x}(k+i-d \mid k),
\end{aligned}
$$

where $\mathbf{K}_{A}(k), \Delta \mathbf{K}_{A}(k), \mathbf{K}_{A d}(k), \mathbf{K}_{M}(k), \Delta \mathbf{K}_{M}(k), \mathbf{K}_{M d}(k)$ are gain matrixes to be determined by RMPC strategies. Assume that $\Delta \mathbf{K}_{A}(k)$ and $\Delta \mathbf{K}_{M}(k)$ have the following forms as in (10) and (11), respectively, representing additive gain perturbation case (referred to as Case I) and multiplicative gain perturbation case (referred to as Case II):

$$
\begin{aligned}
\Delta \mathbf{K}_{A}(k) & =\mathbf{M L}(k) \mathbf{N}, \quad\|\mathbf{L}(k)\|_{2} \leq 1, \\
\Delta \mathbf{K}_{M}(k) & =\mathbf{U W}(k) \mathbf{V} \mathbf{K}_{M}, \quad\|\mathbf{W}(k)\|_{2} \leq 1,
\end{aligned}
$$

where $\mathbf{M}, \mathbf{N}, \mathbf{U}, \mathbf{V}$ are constant matrixes with appropriate dimensions and $\mathbf{L}(k), \mathbf{W}(k)$ can represent any unknown matrixes satisfying norm-bounded conditions by scaling $\mathbf{M}$, $\mathbf{N}, \mathbf{U}$, and $\mathbf{V}$.

For systems (1) subject to constraints, the following infinite horizon quadratic cost function is first defined:

$$
\mathbf{J}(k)=\sum_{i=0}^{\infty}\left[\|\mathbf{x}(k+i \mid k)\|_{\mathbf{Q}_{1}}+\|\mathbf{u}(k+i \mid k)\|_{\mathbf{R}}\right],
$$

where $\mathbf{Q}_{1}$ and $\mathbf{R}$ are given positive definite symmetric weighting matrixes. Consider the following min-max optimization problem, which minimizes the worst case infinite horizon quadratic objective function.

Case I is as follows:

$$
\min _{\mathbf{u}_{A}(k+i \mid k)(0 \leq k \leq \infty)} \max _{\Delta \mathbf{A}(k), \Delta \mathbf{A}_{d}(k), \Delta \mathbf{K}_{A}(k)} \mathbf{J}(k) .
$$

Case II is as follows:

$$
\min _{\mathbf{u}_{M}(k+i \mid k)(0 \leq k \leq \infty)} \max _{\Delta \mathbf{A}(k), \Delta \mathbf{A}_{d}(k), \Delta \mathbf{K}_{M}(k)} \mathbf{J}(k) .
$$

In order to design such controllers as Cases I and II, the upper bound of the cost in (13) or (14) needs to be determined.
According to Lyapunov-Krasovskii function, a delay state dependent quadratic function is chosen as follows:

$$
\mathbf{V}(\mathbf{x}(k))=\|\mathbf{x}(k)\|_{\mathbf{P}}+\sum_{j=1}^{d}\|\mathbf{x}(k-j)\|_{\mathbf{S}},
$$

where $\mathbf{P}=\mathbf{P}^{T}>0$ and $\mathbf{S}=\mathbf{S}^{T}>0$ are positive definite symmetric matrixes and satisfy the following condition to guarantee the cost monotonicity:

$$
\begin{aligned}
\Delta \mathbf{V}(i, k) & =\mathbf{V}(\mathbf{x}(k+i+1 \mid k))-\mathbf{V}(\mathbf{x}(k+i \mid k)) \\
& \leq-\|\mathbf{x}(k+i \mid k)\|_{\mathbf{Q}_{1}}-\|\mathbf{u}(k+i \mid k)\|_{\mathbf{R}} .
\end{aligned}
$$

For the cost in (13) or (14) to be finite, $\mathbf{V}(\mathbf{x}(\infty \mid k))=0$ is assumed. Summing both sides of (16) from $i=0$ to $i=\infty$, the worst value of the cost function $\mathrm{J}(k)$ will be bounded by the following.

Case I is as follows:

$$
\begin{array}{ll}
\max _{\Delta \mathbf{A}(k), \Delta \mathbf{A}_{d}(k), \Delta \mathbf{K}_{A}(k)} & \mathbf{J}(k) \leq \mathbf{V}(\mathbf{x}(k \mid k)) \\
= & \|\mathbf{x}(k \mid k)\|_{\mathbf{P}} \\
& +\sum_{j=1}^{d}\|\mathbf{x}(k-j \mid k)\|_{\mathbf{S}} .
\end{array}
$$

Case II is as follows:

$$
\begin{aligned}
& \max _{\Delta \mathbf{A}(k), \Delta \mathbf{A}_{d}(k), \Delta \mathbf{K}_{M}(k)} \quad \mathbf{J}(k) \leq \mathbf{V}(\mathbf{x}(k \mid k)) \\
& =\|\mathbf{x}(k \mid k)\|_{\mathbf{P}} \\
& \\
& \quad+\sum_{j=1}^{d}\|\mathbf{x}(k-j \mid k)\|_{\mathbf{S}} .
\end{aligned}
$$

Therefore, the above optimization can be reformulated as follows.

Case I is

$$
\begin{array}{ll}
\min _{\mathbf{P}, \mathbf{S}} & {\left[\|\mathbf{x}(k \mid k)\|_{\mathbf{P}}+\sum_{j=1}^{d}\|\mathbf{x}(k-j \mid k)\|_{\mathbf{S}}\right]} \\
\text { s.t. } & (1),(8),(16),(17) .
\end{array}
$$

Case II is

$$
\begin{array}{ll}
\min _{\mathbf{P}, \mathbf{S}} & {\left[\|\mathbf{x}(k \mid k)\|_{\mathbf{P}}+\sum_{j=1}^{d}\|\mathbf{x}(k-j \mid k)\|_{\mathbf{S}}\right]} \\
\text { s.t. } & (1),(9),(16),(18) .
\end{array}
$$

In order to convert the minimization problem in (19) and (20) into LMI optimization problems that can be efficiently solved by convex optimization algorithm, the upper bound of the objective function in (17) and (18) is defined as follows. 
Cases I and II are

$$
\|\mathbf{x}(k \mid k)\|_{\mathbf{P}}+\sum_{j=1}^{d}\|\mathbf{x}(k-j \mid k)\|_{\mathbf{s}} \leq \gamma,
$$

where $\gamma$ is the nonnegative upper bound of $\mathbf{V}(\mathbf{x}(k))$ to be minimized and may be different for Cases I and II. According to (21), the original min-max problem (19) and (20) can be redefined to be the following optimization problem that minimizes an upper bound on the worst value of the original cost function $\mathbf{J}(k)$.

Case I is

$$
\begin{array}{ll}
\min _{\gamma, \mathbf{P}, \mathbf{S}} & \gamma \\
\text { s.t. } & (1),(8),(16),(21) .
\end{array}
$$

Case II is

$$
\begin{array}{ll}
\min _{\gamma, \mathbf{P}, \mathbf{S}} & \gamma \\
\text { s.t. } & (1),(9),(16),(21) .
\end{array}
$$

Remark 4. With the rapid development of computer and automation technology, more and more attention has been paid to digital control system. However, physical limits in available microprocessor memory, errors of truncation and quantization of the A/D and D/A converters, and so forth always cause the controller parameters trivial deviations from the original design values. In recent years, many theories exist for nonfragile or insensitive or resilient robust controllers design for systems with additive and multiplicative gain perturbations. However, only a few MPC algorithms [23] have been published that handle the controller parameters deviations explicitly.

\section{Main Results}

4.1. Additive Gain Perturbation Case. In this section, an approach to design nonfragile RMPC algorithm for uncertain time-delay systems with objective function (12) is presented. First, substituting (8) into (1), one can get its corresponding closed-loop system as

$$
\mathbf{x}(k+1)=\widetilde{\mathbf{A}}(k) \mathbf{x}(k)+\widetilde{\mathbf{A}}_{d}(k) \mathbf{x}(k-d),
$$

where

$$
\begin{aligned}
\widetilde{\mathbf{A}}(k) & =\mathbf{A}+\Delta \mathbf{A}(k)+\mathbf{B}\left(\mathbf{K}_{A}+\Delta \mathbf{K}_{A}(k)\right), \\
\widetilde{\mathbf{A}}_{d}(k) & =\mathbf{A}_{d}+\Delta \mathbf{A}_{d}(k)+\mathbf{B} \mathbf{K}_{A d} .
\end{aligned}
$$

Next, a sufficient condition is given for the existence of nonfragile RMPC algorithm for uncertain time-delay systems with additive gain perturbation.

Theorem 5. For uncertain time-delay systems (1) with both delay compensation and controller gain perturbation described by (8) and (10), if there exist appropriate positive constants $\boldsymbol{\Lambda}_{i}(i=1,2,3,4), \alpha$, matrixes $\mathbf{Y}, \mathbf{Y}_{d}, \Gamma$, and symmetric matrixes $\mathbf{W}>0, \mathbf{Q}>0$, such that the following LMIs hold

$$
\begin{aligned}
& \min _{\gamma, \mathbf{Y}, \mathbf{Y}_{d}, \boldsymbol{\Gamma}, \mathbf{W}, \mathbf{Q}} \gamma \\
& {\left[\begin{array}{ccccccc}
0.5 \mathbf{Q} & * & * & * & * & * & * \\
\mathbf{Q}_{1}^{0.5} \mathbf{Q} & 2 \gamma \mathbf{I} & * & * & * & * & * \\
\mathbf{Y} & \mathbf{0} & 2\left(\boldsymbol{\Gamma}-\boldsymbol{\Lambda}_{2} \mathbf{M M}^{T}\right) & * & * & * & * \\
\mathbf{N Q} & \mathbf{0} & \mathbf{0} & \left(\boldsymbol{\Lambda}_{1}+2 \boldsymbol{\Lambda}_{2}\right) \mathbf{I} & * & * & * \\
\mathbf{F Q} & \mathbf{0} & \mathbf{0} & \mathbf{0} & \boldsymbol{\Lambda}_{3} \mathbf{I} & * & * \\
\mathbf{Q} & \mathbf{0} & \mathbf{0} & \mathbf{0} & \mathbf{0} & 2 \mathbf{W} & * \\
\mathbf{A Q}+\mathbf{B Y} & \mathbf{0} & \mathbf{0} & \mathbf{0} & \mathbf{0} & \mathbf{0} & \mathbf{Q}-\boldsymbol{\Lambda}_{1} \mathbf{B M}^{T} \mathbf{B}^{T}-\boldsymbol{\Lambda}_{3} \mathbf{E E}^{T}
\end{array}\right]>0,} \\
& {\left[\begin{array}{ccc}
\boldsymbol{\Gamma} & * & * \\
\mathbf{M}^{T} & \mathbf{0} & * \\
\mathbf{0} & \mathbf{I} & -\boldsymbol{\Lambda}_{2} \mathbf{I}
\end{array}\right]>0,} \\
& {\left[\begin{array}{ccccc}
\mathbf{Q} & * & * & * & * \\
\mathbf{E}^{T} & \mathbf{0} & * & * & * \\
\mathbf{0} & \mathbf{I} & -\boldsymbol{\Lambda}_{3} \mathbf{I} & * & * \\
\mathbf{M}^{T} \mathbf{B}^{T} & \mathbf{0} & \mathbf{0} & \mathbf{0} & * \\
\mathbf{0} & \mathbf{0} & \mathbf{0} & \mathbf{I} & -\boldsymbol{\Lambda}_{1} \mathbf{I}
\end{array}\right]>0}
\end{aligned}
$$




$$
\begin{aligned}
& {\left[\begin{array}{cccc}
0.5 \mathbf{W} & * & * & * \\
\mathbf{R}^{0.5} \mathbf{Y}_{d} & 2 \gamma \mathbf{I} & * & * \\
\mathbf{G W} & \mathbf{0} & \boldsymbol{\Lambda}_{4} \mathbf{I} & * \\
\mathbf{A}_{d} \mathbf{W}+\mathbf{B} \mathbf{Y}_{d} & \mathbf{0} & \mathbf{0} & \mathbf{Q}-\boldsymbol{\Lambda}_{4} \mathbf{H} \mathbf{H}^{T}
\end{array}\right]>0} \\
& {\left[\begin{array}{ccc}
\mathbf{Q} & * & * \\
\mathbf{H}^{T} & \mathbf{0} & * \\
\mathbf{0} & \mathbf{I} & -\boldsymbol{\Lambda}_{4} \mathbf{I}
\end{array}\right]>0,}
\end{aligned}
$$

$$
\begin{aligned}
& {\left[\begin{array}{ccccc}
1 & * & * & * & * \\
\mathbf{x}(k \mid k) & \mathbf{Q} & * & * & * \\
\mathbf{x}(k-1 \mid k) & \mathbf{0} & \mathbf{W} & * & * \\
\vdots & \mathbf{0} & \mathbf{0} & \ddots & * \\
\mathbf{x}(k-d \mid k) & \mathbf{0} & \mathbf{0} & \mathbf{0} & \mathbf{W}
\end{array}\right]>0,}
\end{aligned}
$$

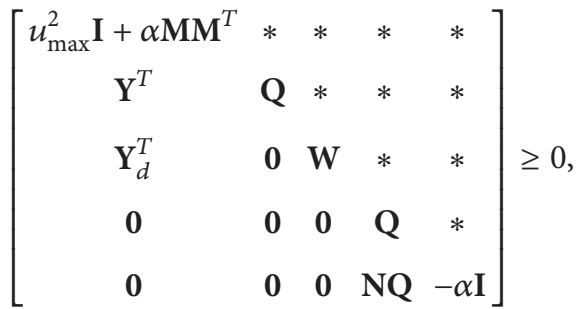

then $\mathbf{u}(k \mid k)=\mathbf{Y Q}^{-1} \mathbf{x}(k \mid k)+\mathbf{Y}_{d} \mathbf{W}^{-1} \mathbf{x}(k-d \mid k)$ is a nonfragile RMPC algorithm satisfying the input constraint described in (1) and the upper bound of $\mathbf{J}(k)$ is less than $\gamma$.

$$
\begin{aligned}
\Delta \mathbf{V}(i, k) & =\mathbf{V}(\mathbf{x}(k+i+1 \mid k))-\mathbf{V}(\mathbf{x}(k+i \mid k)) \\
& =\|\mathbf{x}(k+i+1 \mid k)\|_{\mathbf{P}}+\sum_{j=1}^{d}\|\mathbf{x}(k+i+1-j \mid k)\|_{\mathbf{S}}-\|\mathbf{x}(k+i \mid k)\|_{\mathbf{P}}-\sum_{j=1}^{d}\|\mathbf{x}(k+i-j \mid k)\|_{\mathbf{S}} \\
& =\|\mathbf{x}(k+i+1 \mid k)\|_{\mathbf{P}}-\|\mathbf{x}(k+i \mid k)\|_{\mathbf{P}}+\|\mathbf{x}(k+i \mid k)\|_{\mathbf{S}}-\|\mathbf{x}(k+i-d \mid k)\|_{\mathbf{S}} \\
& =\left\|\tilde{\mathbf{A}}(k) \mathbf{x}(k+i \mid k)+\tilde{\mathbf{A}}_{d}(k) \mathbf{x}(k+i-d \mid k)\right\|_{\mathbf{P}}-\|\mathbf{x}(k+i \mid k)\|_{\mathbf{P}}+\|\mathbf{x}(k+i \mid k)\|_{\mathbf{S}}-\|\mathbf{x}(k+i-d \mid k)\|_{\mathbf{S}} \\
& =\left[\begin{array}{c}
\mathbf{x}(k+i \mid k) \\
\mathbf{x}(k+i-d \mid k)
\end{array}\right]^{T}\left[\begin{array}{cc}
\tilde{\mathbf{A}}^{T}(k) \mathbf{P} \tilde{\mathbf{A}}(k)+\mathbf{S}-\mathbf{P} & * \\
\tilde{\mathbf{A}}_{d}^{T}(k) \mathbf{P} \tilde{\mathbf{A}}(k) & \tilde{\mathbf{A}}_{d}^{T}(k) \mathbf{P} \tilde{\mathbf{A}}_{d}(k)-\mathbf{S}
\end{array}\right]\left[\begin{array}{c}
\mathbf{x}(k+i \mid k) \\
\mathbf{x}(k+i-d \mid k)
\end{array}\right] \\
& \leq-\|\mathbf{x}(k+i \mid k)\|_{\mathbf{Q}_{1}}-\|\mathbf{u}(k+i \mid k)\|_{\mathbf{R}} .
\end{aligned}
$$

Since $\mathbf{u}(k+i \mid k)=\left(\mathbf{K}_{A}+\Delta \mathbf{K}_{A}(k)\right) \mathbf{x}(k+i \mid k)+\mathbf{K}_{A d} \mathbf{x}(k+$ $i-d \mid k)$, the following equation can be obtained directly:

$$
\begin{aligned}
& \|\mathbf{u}(k+i \mid k)\|_{\mathbf{R}}=\mathbf{u}^{T}(k+i \mid k) \mathbf{R} \mathbf{u}(k+i \mid k) \\
& \quad=\left[\begin{array}{c}
\mathbf{x}(k+i \mid k) \\
\mathbf{x}(k+i-d \mid k)
\end{array}\right]^{T} \boldsymbol{\Theta}(k)\left[\begin{array}{c}
\mathbf{x}(k+i \mid k) \\
\mathbf{x}(k+i-d \mid k)
\end{array}\right],
\end{aligned}
$$


where

$\Theta(k)$

$$
=\left[\begin{array}{cc}
\left(\mathbf{K}_{A}+\Delta \mathbf{K}_{A}(k)\right)^{T} \mathbf{R}\left(\mathbf{K}_{A}(k)+\Delta \mathbf{K}_{A}(k)\right) & * \\
\mathbf{K}_{A d}^{T} \mathbf{R}\left(\mathbf{K}_{A}+\Delta \mathbf{K}_{A}(k)\right) & \mathbf{K}_{A d}^{T} \mathbf{R} \mathbf{K}_{A d}
\end{array}\right] .
$$

Combining (34) and (35), it is clear that

$$
\begin{aligned}
& \Delta \mathbf{V}(i, k)+\|\mathbf{x}(k+i \mid k)\|_{\mathbf{Q}_{1}}+\|\mathbf{u}(k+i \mid k)\|_{\mathbf{R}} \\
& \quad=\left[\begin{array}{c}
\mathbf{x}(k+i \mid k) \\
\mathbf{x}(k+i-d \mid k)
\end{array}\right]^{T} \mathbf{M}(k)\left[\begin{array}{c}
\mathbf{x}(k+i \mid k) \\
\mathbf{x}(k+i-d \mid k)
\end{array}\right] \\
& \quad \leq 0,
\end{aligned}
$$

where

$$
\begin{aligned}
& \mathbf{M}(k)=\left[\begin{array}{cc}
\mathbf{M}_{1}(k)+\mathbf{Q}_{1}+\left(\mathbf{K}_{A}+\Delta \mathbf{K}_{A}(k)\right)^{T} \mathbf{R}\left(\mathbf{K}_{A}+\Delta \mathbf{K}_{A}(k)\right) & * \\
\mathbf{M}_{2}(k)+\mathbf{K}_{A d}^{T} \mathbf{R}\left(\mathbf{K}_{A}(k)+\Delta \mathbf{K}_{A}(k)\right) & \mathbf{M}_{3}(k)+\mathbf{K}_{A d}^{T} \mathbf{R} \mathbf{K}_{A d}
\end{array}\right], \\
& \mathbf{M}_{1}(k)=\left(\mathbf{A}+\mathbf{B} \mathbf{K}_{A}+\mathbf{E} \boldsymbol{\Delta}_{1}(k) \mathbf{F}+\mathbf{B} \mathbf{M L}(k) \mathbf{N}\right)^{T} \mathbf{P}\left(\mathbf{A}+\mathbf{B} \mathbf{K}_{A}+\mathbf{E} \boldsymbol{\Delta}_{1}(k) \mathbf{F}+\mathbf{B M L}(k) \mathbf{N}\right)+\mathbf{S}-\mathbf{P}, \\
& \mathbf{M}_{2}(k)=\left(\mathbf{A}_{d}+\mathbf{H} \boldsymbol{\Delta}_{2}(k) \mathbf{G}+\mathbf{B} \mathbf{K}_{A d}\right)^{T} \mathbf{P}\left(\mathbf{A}+\mathbf{B} \mathbf{K}_{A}+\mathbf{E} \boldsymbol{\Delta}_{1}(k) \mathbf{F}+\mathbf{B M L}(k) \mathbf{N}\right), \\
& \mathbf{M}_{3}(k)=\left(\mathbf{A}_{d}+\mathbf{H} \boldsymbol{\Delta}_{2}(k) \mathbf{G}+\mathbf{B} \mathbf{K}_{A d}\right)^{T} \mathbf{P}\left(\mathbf{A}_{d}+\mathbf{H} \boldsymbol{\Delta}_{2}(k) \mathbf{G}+\mathbf{B} \mathbf{K}_{A d}\right)-\mathbf{S} .
\end{aligned}
$$

According to Lemma 1, the following holds:

$$
\begin{aligned}
& \mathbf{x}^{T}(k+i \mid k)\left[\mathbf{K}_{A d}^{T} \mathbf{R}\left(\mathbf{K}_{A}+\Delta \mathbf{K}_{A}(k)\right)\right] \mathbf{x}(k+i-d \mid k) \\
& +\mathbf{x}^{T}(k+i-d \mid k)\left(\mathbf{A}_{d}+\mathbf{H} \mathbf{\Delta}_{2}(k) \mathbf{G}+\mathbf{B} \mathbf{K}_{A d}\right)^{T} \\
& \cdot \mathbf{P}\left(\mathbf{A}+\mathbf{B} \mathbf{K}_{A}+\mathbf{E} \Delta_{1}(k) \mathbf{F}+\mathbf{B M L}(k) \mathbf{N}\right) \mathbf{x}(k \\
& +i \mid k) \leq \frac{1}{2}\left\|\mathbf{K}_{A d} \mathbf{x}(k+i \mid k)\right\|_{\mathbf{R}} \\
& +\frac{1}{2}\left\|\left(\mathbf{K}_{A}+\mathbf{M L}(k) \mathbf{N}\right) \mathbf{x}(k+i-d \mid k)\right\|_{\mathbf{R}} \\
& +\frac{1}{2}\left\|\left(\mathbf{A}_{d}+\mathbf{H} \boldsymbol{\Delta}_{2}(k) \mathbf{G}+\mathbf{B} \mathbf{K}_{A d}\right) \mathbf{x}(k+i-d \mid k)\right\|_{\mathbf{P}} \\
& +\frac{1}{2} \|\left(\mathbf{A}+\mathbf{B} \mathbf{K}_{A}+\mathbf{E} \mathbf{\Delta}_{1}(k) \mathbf{F}+\mathbf{B M L}(k) \mathbf{N}\right) \\
& \cdot \mathbf{x}(k+i \mid k) \|_{\mathbf{P}} .
\end{aligned}
$$

Then, inequality (37) holds if the following inequality holds:

$$
\begin{aligned}
\mathbf{x}^{T}(k+i \mid k)\left\{\mathbf{S}+\mathbf{Q}_{1}-\mathbf{P}+2\left[\mathbf{A}+\mathbf{B} \mathbf{K}_{A}+\mathbf{E} \boldsymbol{\Delta}_{1}(k) \mathbf{F}\right.\right. \\
+\mathbf{B M L}(k) \mathbf{N}]^{T} \\
\cdot \mathbf{P}\left[\mathbf{A}+\mathbf{B} \mathbf{K}_{A}+\mathbf{E} \boldsymbol{\Delta}_{1}(k) \mathbf{F}+\mathbf{B M L}(k) \mathbf{N}\right] \\
\left.+\left(\mathbf{K}_{A}+\mathbf{M L}(k) \mathbf{N}\right)^{T} \mathbf{R}\left(\mathbf{K}_{A}+\mathbf{M L}(k) \mathbf{N}\right)\right\} \mathbf{x}(k+i \mid
\end{aligned}
$$

$$
\begin{aligned}
& k)+\mathbf{x}^{T}(k+i-d \mid k)\left\{2 \left[\mathbf{A}_{d}+\mathbf{H} \boldsymbol{\Delta}_{2}(k) \mathbf{G}\right.\right. \\
& \left.+\mathbf{B} \mathbf{K}_{A d}\right]^{T} \mathbf{P}\left[\mathbf{A}_{d}+\mathbf{H} \boldsymbol{\Delta}_{2}(k) \mathbf{G}+\mathbf{B K}_{A d}\right]+\mathbf{K}_{A d}^{T}(k) \\
& \left.\cdot \mathbf{R} \mathbf{K}_{A d}-\mathbf{S}\right\} \mathbf{x}(k+i-d \mid k)<0 .
\end{aligned}
$$

Hence, through Lemma 2, inequality (40) can be verified straightforwardly if there exist positive real constants $\varepsilon_{1}, \varepsilon_{2}$, $\varepsilon_{3}$, and $\varepsilon_{4}$ that make the following inequalities hold:

$$
\begin{aligned}
2 & \left.\mathbf{A}+\mathbf{B K}_{A}+\mathbf{E} \boldsymbol{\Delta}_{1}(k) \mathbf{F}+\mathbf{B M L}(k) \mathbf{N}\right]^{T} \\
\cdot & \mathbf{P}\left[\mathbf{A}+\mathbf{B} \mathbf{K}_{A}+\mathbf{E} \Delta_{1}(k) \mathbf{F}+\mathbf{B M L}(k) \mathbf{N}\right] \\
+ & \left(\mathbf{K}_{A}+\mathbf{M L}(k) \mathbf{N}\right)^{T} \mathbf{R}\left(\mathbf{K}_{A}+\mathbf{M L}(k) \mathbf{N}\right)+\mathbf{S}+\mathbf{Q}_{1} \\
& -\mathbf{P} \leq 2\left(\mathbf{A}+\mathbf{B} \mathbf{K}_{A}+\mathbf{E} \Delta_{1}(k) \mathbf{F}\right)^{T} \\
& \cdot\left(\mathbf{P}^{-1}-\varepsilon_{1} \mathbf{B M}(\mathbf{B M})^{T}\right)^{-1}\left(\mathbf{A}+\mathbf{B} \mathbf{K}_{A}+\mathbf{E} \boldsymbol{\Delta}_{1}(k) \mathbf{F}\right) \\
& +2 \varepsilon_{1}^{-1} \mathbf{N}^{T} \mathbf{N}+\mathbf{K}_{A}^{T}\left(\mathbf{R}^{-1}-\varepsilon_{2} \mathbf{M} \mathbf{M}^{T}\right)^{-1} \mathbf{K}_{A} \\
& +\varepsilon_{2}^{-1} \mathbf{N}^{T} \mathbf{N}+\mathbf{S}+\mathbf{Q}_{1}-\mathbf{P} \leq 2\left(\mathbf{A}+\mathbf{B} \mathbf{K}_{A}\right)^{T} \\
& \cdot\left(\mathbf{P}^{-1}-\varepsilon_{1} \mathbf{B M}(\mathbf{B M})^{T}-\varepsilon_{3} \mathbf{E E}^{T}\right)^{-1}\left(\mathbf{A}+\mathbf{B} \mathbf{K}_{A}\right) \\
& +2 \varepsilon_{3}^{-1} \mathbf{F}^{T} \mathbf{F}+2 \varepsilon_{1}^{-1} \mathbf{N}^{T} \mathbf{N}+\mathbf{K}_{A}^{T}\left(\mathbf{R}^{-1}-\varepsilon_{2} \mathbf{M} \mathbf{M}^{T}\right)^{-1} \\
& \cdot \mathbf{K}_{A}+\varepsilon_{2}^{-1} \mathbf{N}^{T} \mathbf{N}+\mathbf{S}+\mathbf{Q}_{1}-\mathbf{P}<0 \\
\mathbf{P}^{-1} & -\varepsilon_{1} \mathbf{B} \mathbf{M}(\mathbf{B M})^{T}>0
\end{aligned}
$$




$$
\begin{aligned}
& \mathbf{R}^{-1}-\varepsilon_{2} \mathbf{M} \mathbf{M}^{T}>0 \\
& \mathbf{P}^{-1}-\varepsilon_{1} \mathbf{B} \mathbf{M}(\mathbf{B M})^{T}-\varepsilon_{3} \mathbf{E E}^{T}>0, \\
& 2\left[\mathbf{A}_{d}+\mathbf{H} \mathbf{\Delta}_{2}(k) \mathbf{G}+\mathbf{B} \mathbf{K}_{A d}\right]^{T} \\
& \quad \cdot \mathbf{P}\left[\mathbf{A}_{d}+\mathbf{H} \boldsymbol{\Delta}_{2}(k) \mathbf{G}+\mathbf{B} \mathbf{K}_{A d}\right]+\mathbf{K}_{A d}^{T} \mathbf{R} \mathbf{K}_{A d}-\mathbf{S} \\
& \quad \leq 2\left(\mathbf{A}_{d}+\mathbf{B} \mathbf{K}_{A d}\right)^{T}\left(\mathbf{P}^{-1}-\varepsilon_{4} \mathbf{H} \mathbf{H}^{T}\right)^{-1}\left(\mathbf{A}_{d}+\mathbf{B} \mathbf{K}_{A d}\right) \\
& \quad+2 \varepsilon_{4}^{-1} \mathbf{G}^{T} \mathbf{G}+\mathbf{K}_{A d}^{T} \mathbf{R} \mathbf{K}_{A d}-\mathbf{S}<0 \\
& \mathbf{P}^{-1}-\varepsilon_{4} \mathbf{H} \mathbf{H}^{T}>0 .
\end{aligned}
$$

It should be noted that inequality (44) is more stringent than inequality (42). Thus, in the following context inequality (44) will be reserved while inequality (42) will be discarded. Multiplying both sides of inequality (41) by $\mathbf{Q}^{T}$ and $\gamma^{-1} \mathbf{Q}$, where $\mathbf{Q}$ denotes a symmetric positive definite matrix, it equals the following inequality:

$$
\begin{aligned}
& \left(\mathbf{A Q}+\mathbf{B} \mathbf{K}_{A} \mathbf{Q}\right)^{T}\left(\gamma \mathbf{P}^{-1}-\gamma \varepsilon_{1} \mathbf{B M}(\mathbf{B M})^{T}-\gamma \varepsilon_{3} \mathbf{E E}^{T}\right)^{-1} \\
& \cdot\left(\mathbf{A Q}+\mathbf{B K} \mathbf{K}_{A} \mathbf{Q}\right)+(\mathbf{F Q})^{T} \frac{1}{\gamma \varepsilon_{3}}(\mathbf{F Q})+(\mathbf{N Q})^{T} \\
& \cdot \frac{1}{\gamma \varepsilon_{1}}(\mathbf{N Q})+\frac{1}{2}\left(\mathbf{K}_{A} \mathbf{Q}\right)^{T}\left(\gamma \mathbf{R}^{-1}-\gamma \varepsilon_{2} \mathbf{M} \mathbf{M}^{T}\right)^{-1} \\
& \cdot \mathbf{K}_{A} \mathbf{Q}+(\mathbf{N Q})^{T} \frac{1}{2 \gamma \varepsilon_{2}}(\mathbf{N Q})+\mathbf{Q}^{T} \frac{\mathbf{S}}{2 \gamma} \mathbf{Q}+\mathbf{Q}^{T} \frac{\mathbf{Q}_{1}}{2 \gamma} \mathbf{Q} \\
& -\frac{\mathbf{Q}}{2}<0 .
\end{aligned}
$$

Now, choose $\Lambda_{1}=\gamma \varepsilon_{1}, \Lambda_{2}=\gamma \varepsilon_{2}, \Lambda_{3}=\gamma \varepsilon_{3}, \mathbf{Y}=$ $\mathbf{K}_{A} \mathbf{Q}, \mathbf{Q}=\gamma \mathbf{P}^{-1}, \boldsymbol{\Gamma}=\gamma \mathbf{R}^{-1}, \mathbf{W}=\gamma \mathbf{S}^{-1}$. Then, by applying Schur complement, inequalities (41), (43), and (44) can be, respectively, derived as the following inequalities:

$$
\begin{aligned}
& {\left[\begin{array}{ccccccc}
0.5 \mathbf{Q} & * & * & * & * & * & * \\
\mathbf{Q}_{1}^{0.5} \mathbf{Q} & 2 \gamma \mathbf{I} & * & * & * & * & * \\
\mathbf{Y} & \mathbf{0} & 2\left(\boldsymbol{\Gamma}-\boldsymbol{\Lambda}_{2} \mathbf{M M}^{T}\right) & * & * & * & * \\
\mathbf{N Q} & \mathbf{0} & \mathbf{0} & \left(\boldsymbol{\Lambda}_{1}+2 \boldsymbol{\Lambda}_{2}\right) \mathbf{I} & * & * & * \\
\mathbf{F Q} & \mathbf{0} & \mathbf{0} & \mathbf{0} & \boldsymbol{\Lambda}_{3} \mathbf{I} & * & * \\
\mathbf{Q} & \mathbf{0} & \mathbf{0} & \mathbf{0} & \mathbf{0} & 2 \mathbf{W} & * \\
\mathbf{A Q}+\mathbf{B Y} & \mathbf{0} & \mathbf{0} & \mathbf{0} & \mathbf{0} & \mathbf{0} & \mathbf{Q}-\boldsymbol{\Lambda}_{1} \mathbf{B M M}^{T} \mathbf{B}^{T}-\boldsymbol{\Lambda}_{3} \mathbf{E E}^{T}
\end{array}\right]>0} \\
& {\left[\begin{array}{cc}
\boldsymbol{\Gamma} & * \\
\mathbf{M}^{T} & \boldsymbol{\Lambda}_{2}^{-1} \mathbf{I}
\end{array}\right]>0,} \\
& {\left[\begin{array}{ccc}
\mathbf{Q} & * & * \\
\mathbf{E}^{T} & \boldsymbol{\Lambda}_{3}^{-1} \mathbf{I} & * \\
\mathbf{M}^{T} \mathbf{B}^{T} & \mathbf{0} & \boldsymbol{\Lambda}_{1}^{-1} \mathbf{I}
\end{array}\right]>0 .}
\end{aligned}
$$

By applying Schur complement, inequalities (49) and (50) can be rewritten as inequalities (28) and (29) in Theorem 5, respectively. Multiplying both sides of inequality (45) by $\mathbf{W}^{T}$ and $\gamma^{-1} \mathbf{W}$, it equals the following inequality:

$$
\begin{gathered}
\left(\mathbf{A}_{d} \mathbf{W}+\mathbf{B} \mathbf{K}_{A d} \mathbf{W}\right)^{T}\left(\gamma \mathbf{P}^{-1}-\gamma \varepsilon_{4} \mathbf{H} \mathbf{H}^{T}\right)^{-1} \\
\cdot\left(\mathbf{A}_{d} \mathbf{W}+\mathbf{B} \mathbf{K}_{A d} \mathbf{W}\right)+(\mathbf{G W})^{T} \frac{1}{\gamma \varepsilon_{4}} \mathbf{G W} \\
+\left(\mathbf{K}_{A d} \mathbf{W}\right)^{T} \frac{\mathbf{R}}{2 \gamma} \mathbf{K}_{A d} \mathbf{W}-\frac{1}{2} \mathbf{W}<0 .
\end{gathered}
$$

Now, choose $\boldsymbol{\Lambda}_{4}=\gamma \varepsilon_{4}, \mathbf{Y}_{d}=\mathbf{K}_{A d} \mathbf{W}, \mathbf{Q}=\gamma \mathbf{P}^{-1}$. Then, by applying Schur complement, inequalities (45) and (46) can be derived as the following inequalities:

$$
\begin{gathered}
{\left[\begin{array}{cccc}
0.5 \mathbf{W} & * & * & * \\
\mathbf{R}^{0.5} \mathbf{Y}_{d} & 2 \gamma \mathbf{I} & * & * \\
\mathbf{G W} & \mathbf{0} & \boldsymbol{\Lambda}_{4} \mathbf{I} & * \\
\mathbf{A}_{d} \mathbf{W}+\mathbf{B Y}_{d} & \mathbf{0} & \mathbf{0} & \mathbf{Q}-\boldsymbol{\Lambda}_{4} \mathbf{H} \mathbf{H}^{T}
\end{array}\right]>0} \\
\\
{\left[\begin{array}{cc}
\mathbf{Q} & * \\
\mathbf{H}^{T} & \boldsymbol{\Lambda}_{4}^{-1} \mathbf{I}
\end{array}\right]>0 .}
\end{gathered}
$$


Similarly, inequality (53) can be rewritten as (31) in Theorem 5 based on the Schur complement. Next, we will derive the LMI condition for inequality (21). Multiplying both sides of inequality (21) with $\gamma^{-1}$, one can get

$$
\mathbf{x}^{T}(k) \gamma^{-1} \mathbf{P} \mathbf{x}(k)+\sum_{j=1}^{d} \mathbf{x}^{T}(k-j) \gamma^{-1} \mathbf{S} \mathbf{x}(k-j) \leq 1
$$

Since $\mathbf{Q}=\gamma \mathbf{P}^{-1}, \mathbf{W}=\gamma \mathbf{S}^{-1}$, according to Schur complement, inequality (54) will be satisfied if the following inequality holds:

$$
\left[\begin{array}{ccccc}
1 & * & * & * & * \\
\mathbf{x}(k \mid k) & \mathbf{Q} & * & * & * \\
\mathbf{x}(k-1 \mid k) & \mathbf{0} & \mathbf{W} & * & * \\
\vdots & \mathbf{0} & \mathbf{0} & \ddots & * \\
\mathbf{x}(k-d \mid k) & \mathbf{0} & \mathbf{0} & \mathbf{0} & \mathbf{W}
\end{array}\right]>0 .
$$

The control input constraint in (1) can be converted into LMI condition. From (21), one obtains

$$
\begin{aligned}
\mathbf{x}^{T}(k & +i \mid k) \gamma^{-1} \mathbf{P x}(k+i \mid k) \\
& +\sum_{j=1}^{d} \mathbf{x}^{T}(k+i-j \mid k) \gamma^{-1} \mathbf{S} \mathbf{x}(k+i-j \mid k) \leq 1,
\end{aligned}
$$

$$
i \geq 0 \text {. }
$$

For $\mathbf{Q}=\gamma \mathbf{P}^{-1}>0, \mathbf{W}=\gamma \mathbf{S}^{-1}>0$, one has

$$
\begin{aligned}
\mathbf{x}^{T}(k & +i \mid k) \mathbf{Q} \mathbf{x}(k+i \mid k) \\
& +\sum_{j=1}^{d} \mathbf{x}^{T}(k+i-j \mid k) \mathbf{W} \mathbf{x}(k+i-j \mid k) \leq 1,
\end{aligned}
$$

$i \geq 0$.

Then, one can get

$$
\begin{array}{r}
\mathbf{x}^{T}(k+i \mid k) \mathbf{Q x}(k+i \mid k) \leq 1, \quad i \geq 0, \\
\mathbf{x}^{T}(k+i-d \mid k) \mathbf{W} \mathbf{x}(k+i-d \mid k) \leq 1, \quad i \geq 0 .
\end{array}
$$

From (8), (10), the input constraint can be expressed as

$$
\begin{aligned}
& \|\mathbf{u}(k+i \mid k)\|_{2} \leq u_{\max } \Longleftrightarrow \\
& \|\left(\mathbf{K}_{A}+\mathbf{M L}(k) \mathbf{N}\right) \mathbf{x}(k+i \mid k)
\end{aligned}
$$

$$
\begin{aligned}
& \quad+\mathbf{K}_{A d} \mathbf{x}(k+i-d \mid k) \|_{2} \leq u_{\max } \Longleftarrow \\
& \left\|\left(\mathbf{K}_{A}+\mathbf{M L}(k) \mathbf{N}\right) \mathbf{x}(k+i \mid k)\right\|_{2} \\
& \quad+\left\|\mathbf{K}_{A d} \mathbf{x}(k+i-d \mid k)\right\|_{2} \leq u_{\max } \Longleftarrow \\
& \left\|\mathbf{K}_{A} \mathbf{x}(k+i \mid k)\right\|_{2}+\|\mathbf{M L}(k) \mathbf{N} \mathbf{x}(k+i \mid k)\|_{2} \\
& \quad+\left\|\mathbf{K}_{A d} \mathbf{x}(k+i-d \mid k)\right\|_{2} \leq u_{\max } \Longleftarrow \\
& \left\|\mathbf{Y Q}^{-1} \mathbf{x}(k+i \mid k)\right\|_{2}+\left\|\mathbf{M L}(k) \mathbf{N Q Q} \mathbf{Q}^{-1} \mathbf{x}(k+i \mid k)\right\|_{2} \\
& \quad+\left\|\mathbf{Y}_{d} \mathbf{W}^{-1} \mathbf{x}(k+i-d \mid k)\right\|_{2} \leq u_{\max } \cdot
\end{aligned}
$$

Similar to the introduction of the invariant ellipsoid used in [5], inequality (59) can be obtained if the following inequality holds:

$$
\left[\begin{array}{cccc}
u_{\max }^{2} \mathbf{I} & * & * & * \\
\mathbf{Y}^{T} & \mathbf{Q} & * & * \\
\mathbf{Y}_{d}^{T} & \mathbf{0} & \mathbf{W} & * \\
(\mathbf{M L}(k) \mathbf{N Q})^{T} & \mathbf{0} & \mathbf{0} & \mathbf{Q}
\end{array}\right] \geq 0 .
$$

Inequality (60) can be rewritten equivalently as

$$
\begin{gathered}
{\left[\begin{array}{cccc}
u_{\max }^{2} \mathbf{I} & * & * & * \\
\mathbf{Y}^{T} & \mathbf{Q} & * & * \\
\mathbf{Y}_{d}^{T} & \mathbf{0} & \mathbf{W} & * \\
\mathbf{0} & \mathbf{0} & \mathbf{0} & \mathbf{Q}
\end{array}\right]+\left[\begin{array}{c}
\mathbf{M} \\
\mathbf{0} \\
\mathbf{0} \\
\mathbf{0}
\end{array}\right] \mathbf{L}(k)\left[\begin{array}{llll}
\mathbf{0} & \mathbf{0} & \mathbf{0} & \mathbf{N Q}
\end{array}\right]} \\
+\left[\begin{array}{c}
\mathbf{0} \\
\mathbf{0} \\
\mathbf{0} \\
(\mathbf{N Q})^{T}
\end{array}\right] \mathbf{L}^{T}(k)\left[\begin{array}{llll}
\mathbf{M}^{T} & \mathbf{0} & \mathbf{0} & \mathbf{0}
\end{array}\right] \geq 0
\end{gathered}
$$

Then, by Lemma 3, inequality (61) holds if there exists a constant $\alpha>0$ such that

$$
\left[\begin{array}{cccc}
u_{\max }^{2} \mathbf{I}+\alpha \mathbf{M M}^{T} & * & * & * \\
\mathbf{Y}^{T} & \mathbf{Q} & * & * \\
\mathbf{Y}_{d}^{T} & \mathbf{0} & \mathbf{W} & * \\
\mathbf{0} & \mathbf{0} & \mathbf{0} & \mathbf{Q}+\alpha^{-1}(\mathbf{N Q})^{T} \mathbf{N Q}
\end{array}\right] \geq 0 .
$$


According to Schur complement, inequality (62) is equivalent to (33) in Theorem 5. This completes the proof.

Remark 6. Strictly speaking, the variables in the above optimization should be denoted by $\mathbf{Y}_{k}, \mathbf{Y}_{d k}, \boldsymbol{\Gamma}_{k}, \mathbf{W}_{k}, \mathbf{Q}_{k}$, and so forth to emphasize that they are computed at time instant $k$. For notational convenience, the subscript is omitted here and in the next section. However, this notation will be briefly used in the robust stability proof (Theorems 7 and 10).

Theorem 7 (robust stability). If the optimization problem (26) is feasible at the initial instant $k=0$, then the proposed nonfragile RMPC law (8) will stabilize system (1) asymptotically.

Proof. Let $\mathbf{P}(k)$ and $\mathbf{P}(k+1)$ denote the optimal solutions of the optimization problem (26) at time instants $k$ and $k+1$, respectively. Let us consider a quadratic function

$$
\mathbf{V}(\mathbf{x}(k))=\|\mathbf{x}(k)\|_{\mathbf{P}}+\sum_{j=1}^{d}\|\mathbf{x}(k-j)\|_{\mathbf{S}}
$$

Since $\mathbf{P}(k+1)$ is optimal and $\mathbf{P}(k)$ is only feasible at time instant $k+1$, one has the following inequality from optimality:

$$
\begin{aligned}
\mathbf{V}(\mathbf{x}(k+1)) & =\|\mathbf{x}(k+1)\|_{\mathbf{P}(k+1)}+\sum_{j=1}^{d}\|\mathbf{x}(k+1-j)\|_{\mathbf{S}} \\
& \leq\|\mathbf{x}(k+1)\|_{\mathbf{P}(k)}+\sum_{j=1}^{d}\|\mathbf{x}(k+1-j)\|_{\mathbf{S}} .
\end{aligned}
$$

Besides, it follows from (16) that

$$
\begin{gathered}
\|\mathbf{x}(k+1 \mid k)\|_{\mathbf{P}(k)}+\sum_{j=1}^{d}\|\mathbf{x}(k+1-j \mid k)\|_{\mathbf{S}} \\
\leq\|\mathbf{x}(k \mid k)\|_{\mathbf{P}(k)}+\sum_{j=1}^{d}\|\mathbf{x}(k-j \mid k)\|_{\mathbf{S}} \\
-\|\mathbf{x}(k \mid k)\|_{\mathbf{Q}_{1}}-\|\mathbf{u}(k \mid k)\|_{\mathbf{R}}
\end{gathered}
$$

for any structured uncertainties $\left[\begin{array}{ll}\Delta \mathbf{A} & \Delta \mathbf{A}_{d}\end{array}\right]$.

Since $\mathbf{x}(k+1 \mid k+1)$ equals $\mathbf{A x}(k \mid k)+\mathbf{B u}(k \mid k)+$ $\mathbf{A}_{d} \mathbf{x}(k-d \mid k)$ for given uncertainties, (65) must hold with $\mathbf{x}(k+1 \mid k+1)$ in place of $\mathbf{x}(k+1 \mid k)$. Combining this with (64), one has

$$
\begin{aligned}
\mathbf{V}(\mathbf{x}(k+1 \mid k+1)) \leq & \mathbf{V}(\mathbf{x}(k \mid k))-\|\mathbf{x}(k \mid k)\|_{\mathbf{Q}_{1}} \\
& -\|\mathbf{u}(k \mid k)\|_{\mathbf{R}} .
\end{aligned}
$$

Summing inequality (66) from $k=0$ to $k=i-1$ yields

$$
\begin{aligned}
& \mathbf{V}(\mathbf{x}(i \mid i))+\sum_{k=0}^{i-1}\|\mathbf{x}(k \mid k)\|_{\mathbf{Q}_{1}}+\|\mathbf{u}(k \mid k)\|_{\mathbf{R}} \\
& \quad \leq \mathbf{V}(\mathbf{x}(0 \mid 0)) .
\end{aligned}
$$

Since $\mathbf{V}(\mathbf{x}(i \mid i)) \geq 0$ and the left side of (67) is bounded by the constant $\mathbf{V}(\mathbf{x}(0 \mid 0))$ and $\mathbf{V}(\mathbf{x}(i \mid i))$ is monotonically nonincreasing, $\mathbf{x}(i \mid i)$ and $\mathbf{u}(i \mid i)$ must converge to zero as $i$ approaches $\infty$. Therefore, the closed-loop stability is guaranteed. Then, the proof is completed.

Remark 8 . The differences between the multiplicative and additive gain perturbations are due to the existence of the former; stability for the systems can be undermined. Moreover, nonlinearity property is presented. As a result, the control problem with multiplicative gain perturbation is more complicated than additive gain perturbation (e.g., linear quadratic Gaussian (LQG) problem). Some issues in stabilization and optimal control problems for such systems are still open for discussion.

4.2. Multiplicative Gain Perturbation Case. In the above section, a sufficient condition to ensure the asymptotic stability of the robust model predictive control with additive gain perturbations is discussed. Next, an approach to design nonfragile RMPC algorithm for uncertain time-delay systems with objective function (12) is presented. By substituting (9) into (1), the corresponding closed-loop system will be obtained as

$$
\mathbf{x}(k+1)=\widetilde{\mathbf{A}}_{M}(k) \mathbf{x}(k)+\widetilde{\mathbf{A}}_{M d}(k) \mathbf{x}(k-d),
$$

where

$$
\begin{aligned}
\widetilde{\mathbf{A}}_{M}(k) & =\mathbf{A}+\Delta \mathbf{A}(k)+\mathbf{B}\left(\mathbf{K}_{M}+\Delta \mathbf{K}_{M}(k)\right), \\
\widetilde{\mathbf{A}}_{M d}(k) & =\mathbf{A}_{d}+\Delta \mathbf{A}_{d}(k)+\mathbf{B} \mathbf{K}_{M d} .
\end{aligned}
$$

Theorem 9. For uncertain time-delay systems (1) with both delay compensation and controller gain perturbation described by (9) and (11), if there exist appropriate positive constants $\Lambda_{i}(i=5,6,7,8), \beta$, matrixes $\mathbf{Y}_{M}, \mathbf{Y}_{M d}, \boldsymbol{\varphi}$, and symmetric matrixes $\mathbf{\Xi}>0, \mathbf{Q}>0$, such that the following LMIs hold 


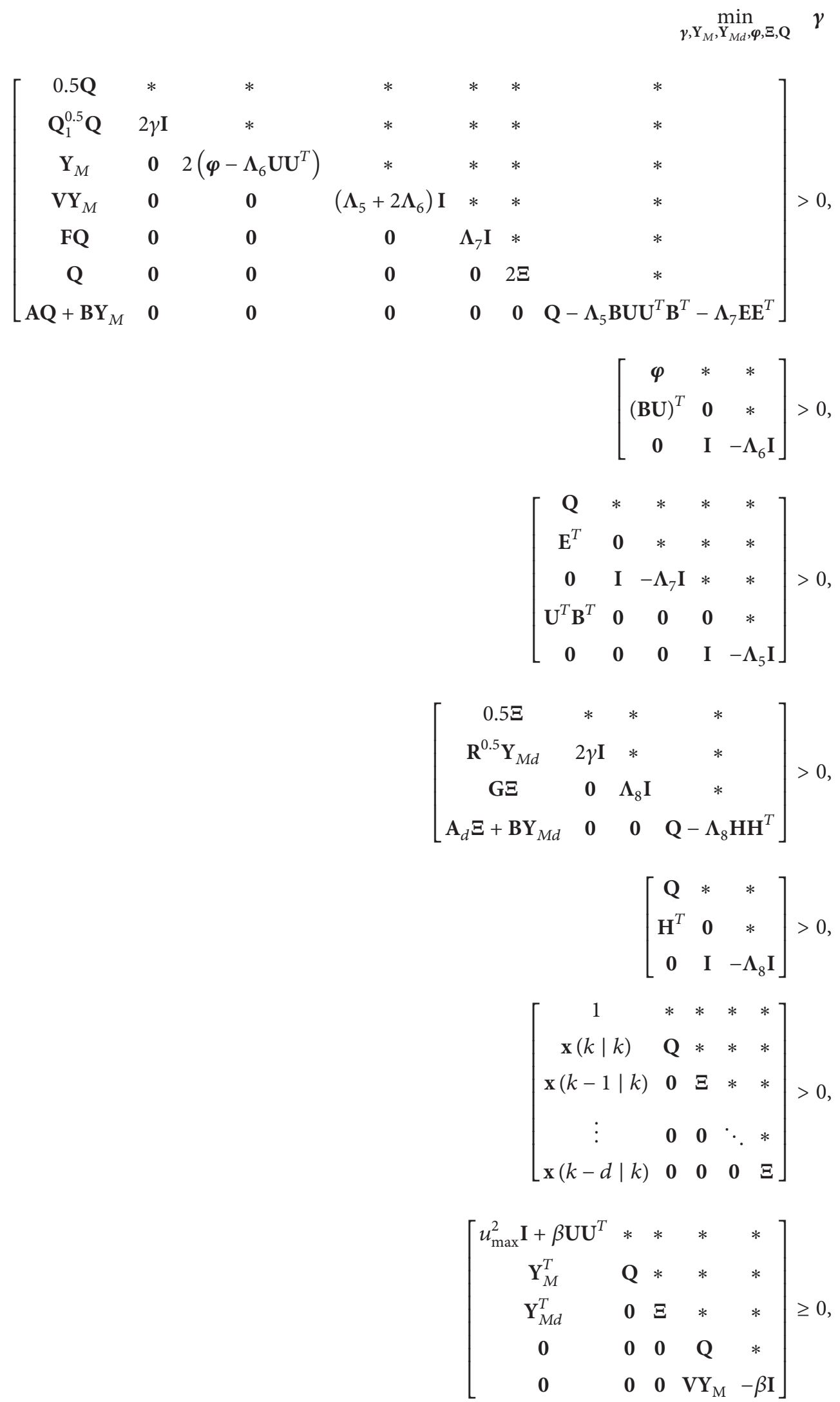


then $\mathbf{u}(k \mid k)=\mathbf{Y}_{M} \mathbf{Q}^{-1} \mathbf{x}(k \mid k)+\mathbf{Y}_{M d} \boldsymbol{\Xi}^{-1} \mathbf{x}(k-d \mid k)$ will be a nonfragile RMPC scheme satisfying input constraint described by (1) and the upper bound of $\mathbf{J}(k)$ will be less than $\boldsymbol{\gamma}$.

Proof. The proof is essentially similar to that of Theorem 5 and thus is omitted due to space limitation.

Theorem 10 (robust stability). If the optimization problem (70) is feasible at the initial instant $k=0$, then the proposed nonfragile RMPC law (9) will stabilize system (1) asymptotically.

Proof. The proof is essentially similar to that of Theorem 7 and thus is omitted due to space limitation.

Remark 11. In the absence of either additive gain perturbation or multiplicative gain perturbation, the result reduces to that in [12]. This corresponds to setting $\mathbf{M}=\mathbf{N}=\mathbf{0}$ (for Case I) and $\mathbf{U}=\mathbf{V}=\mathbf{0}$ (for Case II), respectively. This means that the standard approach is more restrictive and consequently, the algorithms proposed here are less conservative than the one in [12].

Remark 12. The designed nonfragile RMPC algorithms in Theorems 5 and 9 can be extended to a class of discrete-time uncertain linear systems with multiple time-delayed states described as follows:

$$
\begin{aligned}
\mathbf{x}(k+1)= & (\mathbf{A}+\Delta \mathbf{A}(k)) \mathbf{x}(k)+\mathbf{B u}(k) \\
& +\sum_{\tau=1}^{l}\left(\mathbf{A}_{d_{\tau}}+\Delta \mathbf{A}_{d_{\tau}}(k)\right) \mathbf{x}\left(k-d_{\tau}\right),
\end{aligned}
$$

where $d_{\tau}>0(\tau=1,2, \ldots, l)$ denotes certain or uncertain delays and the structured uncertainties $\Delta \mathbf{A}_{d_{\tau}}(k)$ $(\tau=1,2, \ldots, l)$ are time-varying matrixes satisfying normbounded conditions similar to (2). In this case, the corresponding delay-dependent state feedback controller with multiple time-delay compensation can be represented in the following form:

$$
\begin{aligned}
\mathbf{u}_{A / M}(k+i \mid k)= & \left(\mathbf{K}_{A / M}+\Delta \mathbf{K}_{A / M}(k)\right) \mathbf{x}(k+i \mid k) \\
& +\sum_{\tau=1}^{l} \mathbf{K}_{A d_{\tau} / M d_{\tau}} \mathbf{x}\left(k+i-d_{\tau} \mid k\right),
\end{aligned}
$$

where $\mathbf{K}_{A / M}$ and $\mathbf{K}_{A d_{\tau} / M d_{\tau}}(\tau=1,2, \ldots, l)$ denote the gain matrixes to be determined by MPC strategies, respectively, for additive gain perturbation or multiplicative gain perturbation. Similar to the proof of Theorems 5 and 9, corresponding nonfragile RMPC algorithms with multiple time-delay compensation can be derived directly, which are omitted in this paper for the sake of brevity.

Remark 13. Referring to the concept of the asymptotically stable invariant ellipsoid [17] or polyhedral invariant sets [19], the efficient off-line formulation of nonfragile RMPC algorithms with time-delay compensation can be derived directly, which are omitted in this paper for the sake of brevity.

\section{Numerical Simulations}

In this section, two examples will be presented that demonstrate the theoretical results given in previous sections.

Case 1 (additive gain perturbation). Using the same notions mentioned above, let us consider the following discrete-timedelay uncertain linear system as follows:

$$
\begin{aligned}
& \mathbf{A}=\left[\begin{array}{cc}
-0.3 & 1 \\
-0.8 & -0.4
\end{array}\right], \\
& \mathbf{A}_{d}=\left[\begin{array}{cc}
-0.28 & 0.3 \\
0.12 & 0.23
\end{array}\right] \text {, } \\
& \mathbf{B}=\left[\begin{array}{l}
2 \\
1
\end{array}\right], \\
& \mathbf{E}=\left[\begin{array}{c}
0.1 \\
0.22
\end{array}\right] \text {, } \\
& \mathbf{H}=\left[\begin{array}{l}
0.21 \\
0.06
\end{array}\right] \text {, } \\
& \mathbf{F}=\left[\begin{array}{c}
-0.04 \\
0.2
\end{array}\right]^{T} \\
& \mathbf{G}=\left[\begin{array}{c}
-0.1 \\
-0.34
\end{array}\right]^{T} \text {, } \\
& \mathbf{Q}_{1}=\left[\begin{array}{ll}
1 & 0 \\
0 & 1
\end{array}\right] \text {, } \\
& \mathbf{R}=1, \\
& d=2 \text {, } \\
& \boldsymbol{\vartheta}(0)=\left[\begin{array}{c}
0.3 \\
-0.4
\end{array}\right], \\
& \mathbf{x}(0)=\left[\begin{array}{c}
2 \\
-3
\end{array}\right], \\
& \Delta_{1}(k)=\sin (k), \\
& \Delta_{2}(k)=\cos (k) \text {. }
\end{aligned}
$$

The input constraints are

$$
\|\mathbf{u}(k)\|_{2} \leq 2 .
$$

The controller gain perturbation is assumed to have the following form:

$$
\Delta \mathbf{K}=0.05 \sin (t)\left[\begin{array}{ll}
0.8 & -0.4
\end{array}\right] .
$$

All the parameters are substituted to Theorem 5 and the optimization problem of LMIs is solved. The simulation results are compared with the results obtained through 

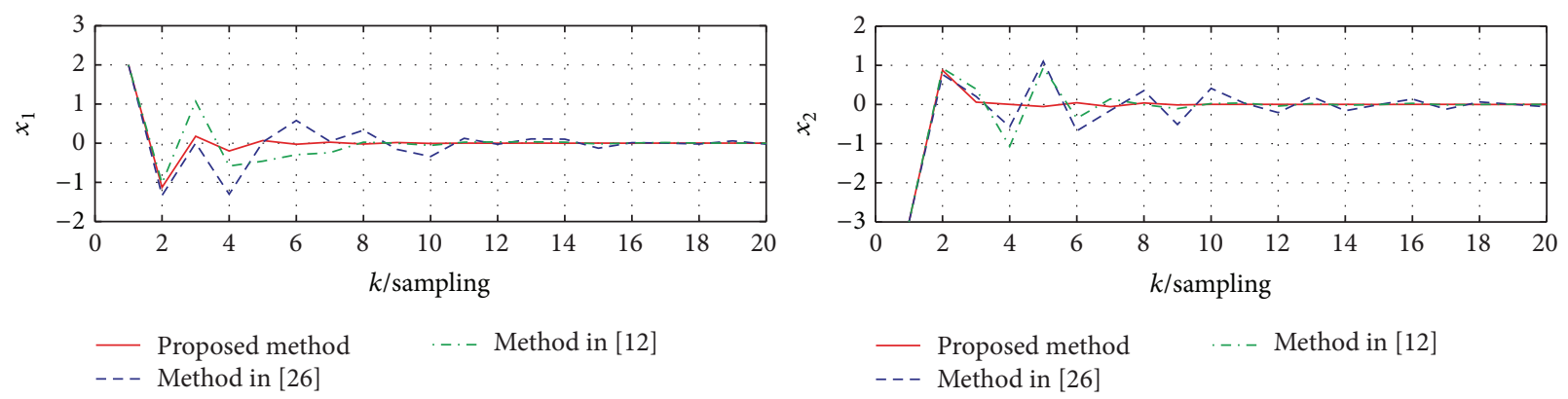

FIGURE 1: State responses for $x_{1}$ and $x_{2}$.
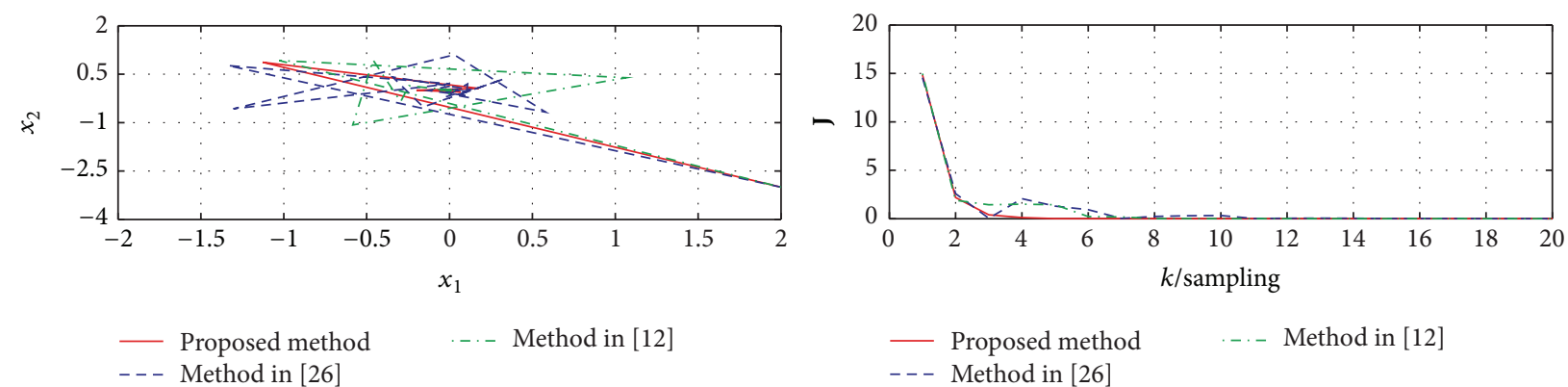

FIGURE 2: State trajectories and cost index J.
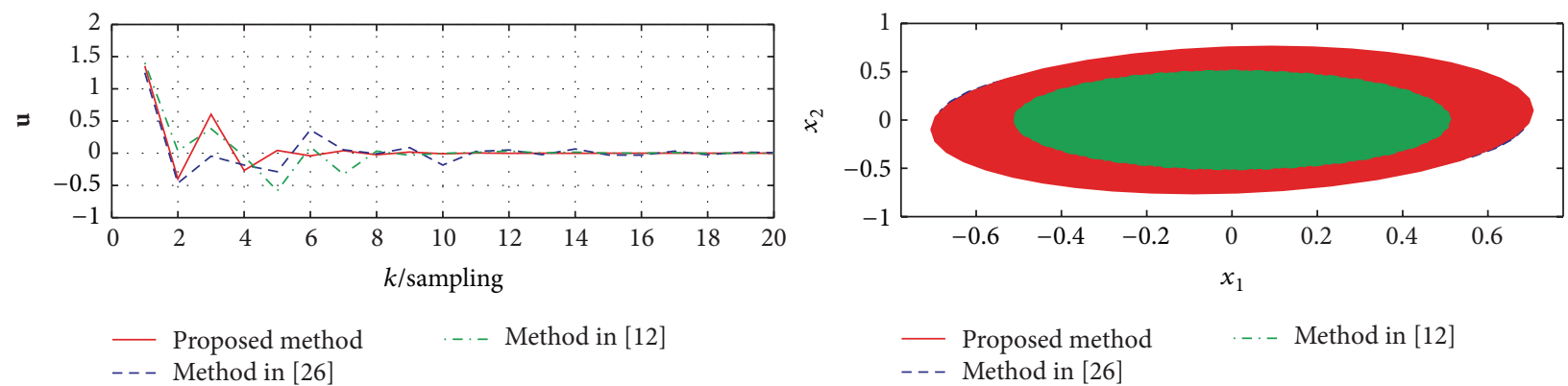

FIgURE 3: Control input and state-invariant ellipsoid $\xi$.

Zhang's method without both gain perturbation and delay compensation in [26] and the results obtained through the method in [12] with merely delay compensation. In the sequence, results obtained through the proposed method in Theorem 5, the method in [26], and the method in [12] are depicted, respectively, by red solid line, blue dashed line, and green dotted line.

The state responses for $x_{1}$ and $x_{2}$ are demonstrated in Figure 1. It shows that all the states through different methods converge to zero. It is obvious that the closed-loop system is stable and the proposed method is superior to the other two methods in convergence speed. Figure 2 shows the state trajectories of the closed-loop system and the cost index with different methods. The cost index of the proposed method is smaller than the other two methods with gain perturbation. The control input and the state-invariant ellipsoid $\boldsymbol{\xi}, \boldsymbol{\xi}=\{\mathbf{z} \mid$ $\left.\mathbf{z}^{T} \mathbf{Q}^{-1} \mathbf{z} \leq 1\right\}$ [5] are shown in Figure 3. According to Figure 3, the inputs are all within their limits and no saturation is observed all the time. Moreover, the state-invariant ellipsoid of the proposed method is the largest. It inclines us to yield a substantial expansion of the region stabilized and achieves a less conservative result. In a word, the improvement is evident in Figures 1 and 3.

Case 2 (multiplicative gain perturbation). Using the same notions mentioned above, we consider the same system in Case 1 with the exception that the controller gain perturbation is assumed to have the following form:

$$
\Delta \mathbf{K}=\left[\begin{array}{ll}
0.05 & 0.1
\end{array}\right] \sin (k)\left[\begin{array}{c}
0.8 \\
-0.4
\end{array}\right] \mathbf{K}
$$

where $\mathbf{K}$ represent $\mathbf{K}_{M}$ in (11). However, the control objective has been modified into tracking a predefined reference 


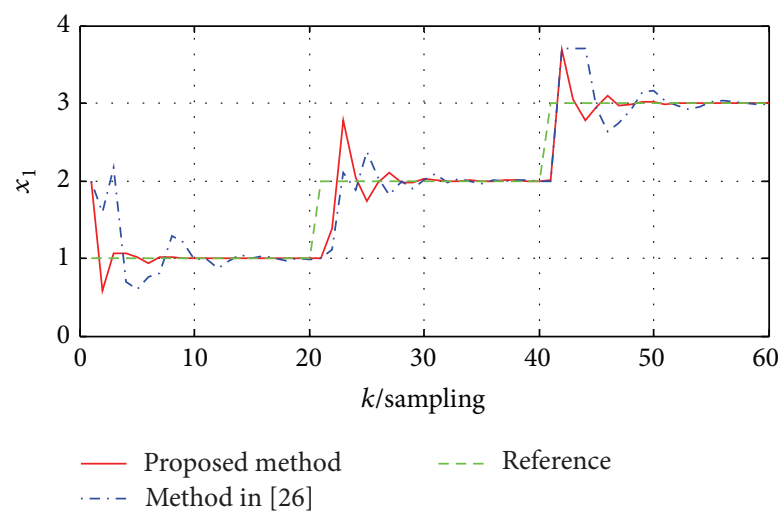

FIGURE 4: Tracking trajectory for $x_{1}$.

trajectory. The benchmark reference trajectory $\aleph_{r}$ is chosen as follows, which is a time-varying piecewise step signal:

$$
\begin{aligned}
& \aleph_{r}: \\
& x_{1}= \begin{cases}1, & 1 \leq k \leq 20, \\
2, & 21 \leq k \leq 40, \\
3, & 41 \leq k \leq 60 ;\end{cases} \\
& x_{2}= \begin{cases}-2, & 1 \leq k \leq 20, \\
-1, & 21 \leq k \leq 40, \\
-2, & 41 \leq k \leq 60 .\end{cases}
\end{aligned}
$$

Similar to Case 1, all the parameters are substituted to Theorem 9 and the optimization problem of LMIs is solved. The simulation results are compared with the results that were obtained through Zhang's method without both gain perturbation and delay compensation in [26]. In the sequence, results obtained through the proposed method in Theorem 9 and the method in [26] are depicted by red solid line and blue dashed line, respectively. The reference tracking performance for $x_{1}$ and $x_{2}$ achieved through the above two methods are, respectively, demonstrated in Figures 4 and 5. The control input is shown in Figure 6. From Figures 4 and 5 , it is clear that the designed controller guarantees global stability and improves the reference tracking performance. Since the input constraints are taken into consideration, thus all the control moves are inside the boundary values as depicted in Figure 6.

From Figures 4, 5, and 6, one can see that the introduction of nonfragility and time-delay compensation can improve the control performance significantly. The above two examples illustrate that the nonfragile RMPC controllers in this paper improve control performance effectively and are more realistic from a practical point of view. All the simulations have been verified on a PC with an Intel Core i3 processor (speed $3.30 \mathrm{GHz}, \mathrm{RAM} 4.00 \mathrm{~GB}$ ) with the software LMI Control Toolbox in the MATLAB environment to compute the solution of the linear minimization problem.



FIGURE 5: Tracking trajectory for $x_{2}$.

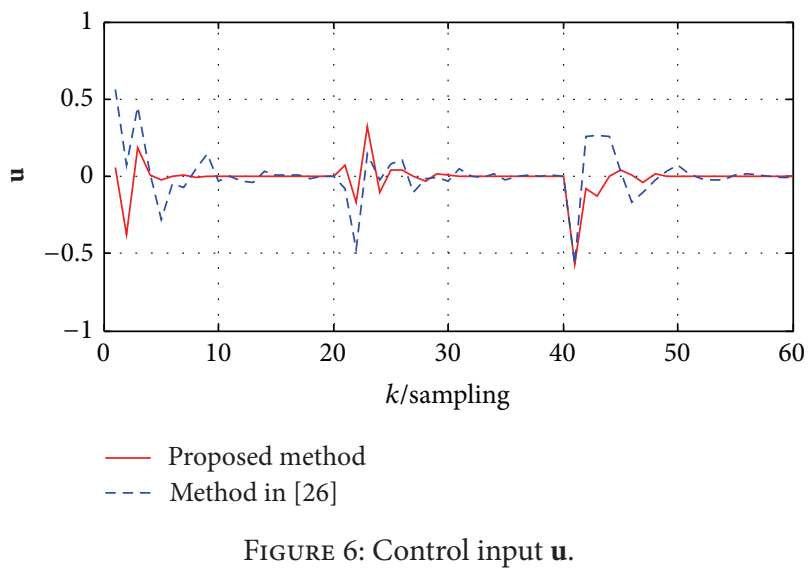

\section{Conclusions}

In this paper, an online nonfragile RMPC algorithm with guaranteed robust stability has been presented for a class of discrete-time structured uncertain time-delayed systems subject to two kinds of gain perturbations, respectively. This method takes into consideration constraints on the inputs and time delay on the states by imposing standard min-max optimization strategy and thus extends the work in $[13,25]$. The development is based on full state feedback assumption and the online optimization involves the solution of a finite dimensional LMI-based linear objective minimization. The numerical example has verified the effectiveness of the proposed designs.

As a further research, the nonfragile output feedback RMPC will be developed to cope with cases where states are partially or totally unavailable. Extension of the results of this paper to more general uncertain systems with both certain/uncertain state and input delays and design of delaydependent RMPC algorithms will be included in the future research work of the authors. Moreover, our future research work on this topic would also be finding other knobs to tackle the LPV model, norm-bounded noise, and one or more free control moves to reduce conservativeness. 


\section{Competing Interests}

The authors declare that there is no conflict of interests regarding the publication of this paper.

\section{Acknowledgments}

The research is partially supported by the Young Scientists Fund of the National Natural Science Foundation of China (Grant nos. 61203007, 61304239, 61503391, and 61503392) and the Natural Science Foundation of Shaanxi Province, China (Grant no. 2013JM8045, 2015JQ6213). Our acknowledgement also goes to Professor Caisheng Jiang for his professional and efficient refinement of this paper.

\section{References}

[1] D. Q. Mayne, J. B. Rawlings, C. V. Rao, and P. O. Scokaert, "Constrained model predictive control: stability and optimality," Automatica, vol. 36, no. 6, pp. 789-814, 2000.

[2] D. Q. Mayne, "Model predictive control: recent developments and future promise," Automatica, vol. 50, no. 12, pp. 2967-2986, 2014.

[3] Y. G. Xi and D. W. Li, "Fundamental philosophy and status of qualitative synthesis of model predictive control," Acta Automatica Sinica, vol. 34, no. 10, pp. 1225-1234, 2008.

[4] Y. G. Xi, D. W. Li, and S. Lin, "Model predictive control—current status and challenges," Acta Automatica Sinica, vol. 39, no. 3, pp. 222-236, 2013.

[5] M. V. Kothare, V. Balakrishnan, and M. Morari, "Robust constrained model predictive control using linear matrix inequalities," Automatica, vol. 32, no. 10, pp. 1361-1379, 1996.

[6] S. C. Jeong and P. Park, "Constrained MPC algorithm for uncertain time-varying systems with state-delay," IEEE Transactions on Automatic Control, vol. 50, no. 2, pp. 257-263, 2005.

[7] M. Lu and H. H. Shao, "Robust predictive control of polytopic uncertain systems with both state and input delays," Journal of Systems Engineering and Electronics, vol. 18, no. 2, pp. 616-621, 2007.

[8] D. H. Ji, J. H. Park, W. J. Yoo, and S. C. Won, "Robust memory state feedback model predictive control for discretetime uncertain state delayed systems," Applied Mathematics and Computation, vol. 215, no. 6, pp. 2035-2044, 2009.

[9] B. Ding and B. Huang, "Constrained robust model predictive control for time-delay systems with polytopic description," International Journal of Control, vol. 80, no. 4, pp. 509-522, 2007.

[10] B. Ding, L. Xie, and W. Cai, "Robust MPC for polytopic uncertain systems with time-varying delays," International Journal of Control, vol. 81, no. 8, pp. 1239-1252, 2008.

[11] Q.-X. Chen, D.-F. He, and L. Yu, "Input-to-state stability of minmax MPC scheme for nonlinear time-varying delay systems," Asian Journal of Control, vol. 14, no. 2, pp. 489-501, 2012.

[12] W.-W. Qin, G. Liu, L.-X. Wang, and Z.-Q. Zheng, "Memory state feedback RMPC for multiple time-delayed uncertain linear systems with input constraints," Mathematical Problems in Engineering, vol. 2014, Article ID 409863, 9 pages, 2014.

[13] W. W. Qin, B. He, P. T. Zhao, and G. Liu, "An improved memory state feedback RMPC for uncertain constrained linear systems with multiple uncertain delays," Mathematical Problems in Engineering, vol. 2015, Article ID 659176, 15 pages, 2015.
[14] Y. Lu and Y. Arkun, "Quasi-min-max MPC algorithms for LPV systems," Automatica, vol. 36, no. 4, pp. 527-540, 2000.

[15] F. A. Cuzzola, J. C. Geromel, and M. Morari, "An improved approach for constrained robust model predictive control," Automatica, vol. 38, no. 7, pp. 1183-1189, 2002.

[16] N. Wada, K. Saito, and M. Saeki, "Model predictive control for linear parameter varying systems using parameter dependent Lyapunov function," IEEE Transactions on Circuits and Systems II: Express Briefs, vol. 53, no. 12, pp. 1446-1450, 2006.

[17] Z. Wan and M. V. Kothare, "An efficient off-line formulation of robust model predictive control using linear matrix inequalities," Automatica, vol. 39, no. 5, pp. 837-846, 2003.

[18] Z. Y. Wan and M. V. Kothare, "Efficient robust constrained model predictive control with a time varying terminal constraint set," Systems \& Control Letters, vol. 48, no. 5, pp. 375-383, 2003.

[19] M.-H. Wang, G. Liu, P.-T. Zhao, and S.-H. Yang, "RMPC algorithm for polytopic constrained LPV systems using polyhedral invariant sets," Control and Decision, vol. 28, no. 11, pp. 16611666, 2013.

[20] S. Wo, Y. Zou, Q. Chen, and S. Xu, "Non-fragile controller design for discrete descriptor systems," Journal of the Franklin Institute, vol. 346, no. 9, pp. 914-922, 2009.

[21] Y. Huang, C. Sun, C. Qian, and L. Wang, "Non-fragile switching tracking control for a flexible air-breathing hypersonic vehicle based on polytopic LPV model," Chinese Journal of Aeronautics, vol. 26, no. 4, pp. 948-959, 2013.

[22] L. Wu, X. Yang, and F. Li, "Nonfragile output tracking control of hypersonic air-breathing vehicles with an LPV model," IEEE/ASME Transactions on Mechatronics, vol. 18, no. 4, pp. 1280-1288, 2013.

[23] Z. Qunliang and X. Yugeng, "Resilient predictive control for a class of uncertain time-delay systems," Journal of Systems Engineering and Electronics, vol. 18, no. 2, pp. 334-340, 2007.

[24] P. Gahinet, A. Nemirovski, A. J. Laub et al., LMI Control Toolbox Users Guide, The Math work Inc, Natick, Mass, USA, 1995.

[25] Y. Y. Wang, L. H. Xie, and C. E. de Souza, "Robust control of a class of uncertain nonlinear systems," Systems \& Control Letters, vol. 19, no. 2, pp. 139-149, 1992.

[26] J. Zhang, R. Pei, X.-Z. Pei, and Z.-Y. Liu, "Robust model for predictive control of uncertain systems with time-delay," Proceedings of the CSEE, vol. 23, no. 7, pp. 212-215, 2003. 


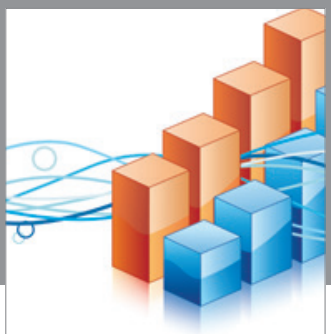

Advances in

Operations Research

vatem alat4

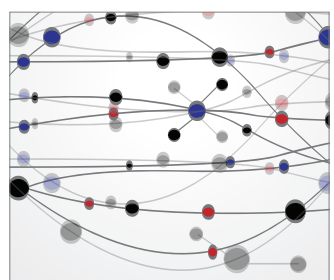

\section{The Scientific} World Journal
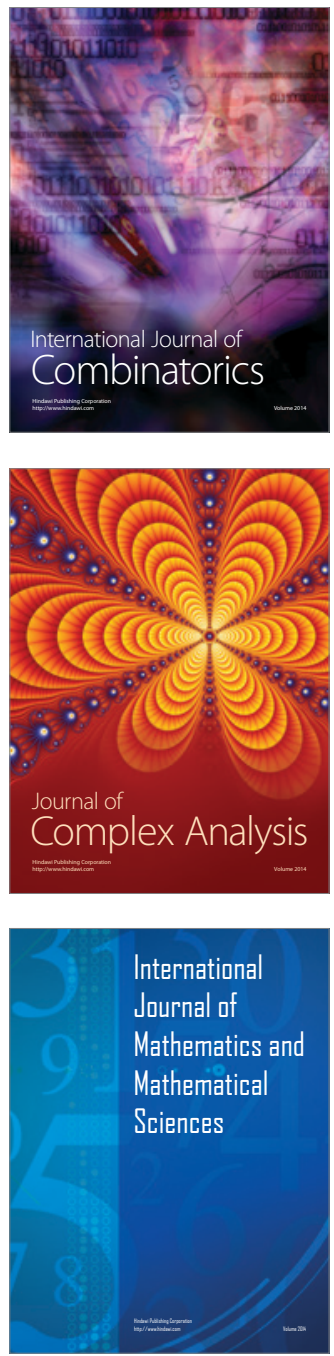


Algebra

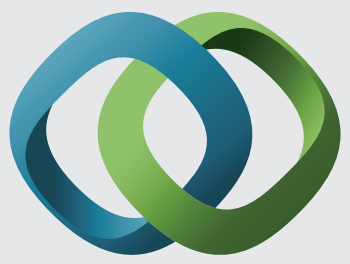

\section{Hindawi}

Submit your manuscripts at

http://www.hindawi.com
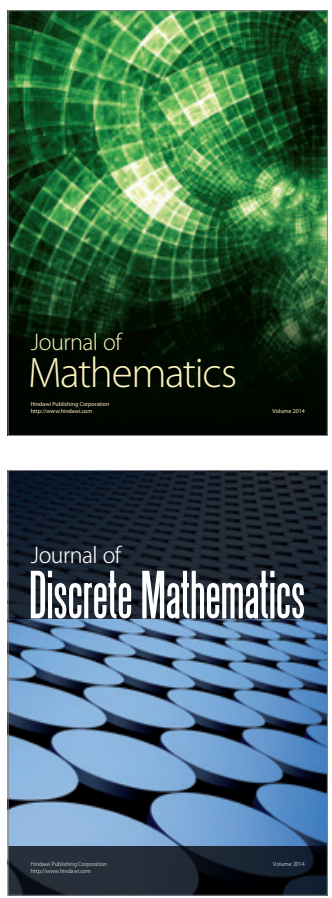



Mathematical Problems in Engineering


Journal of

Function Spaces

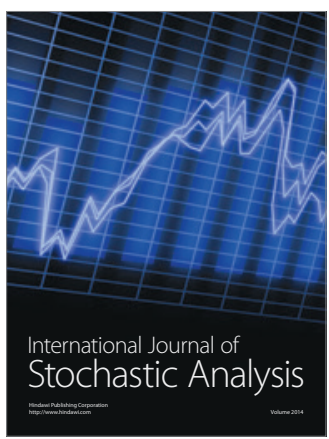

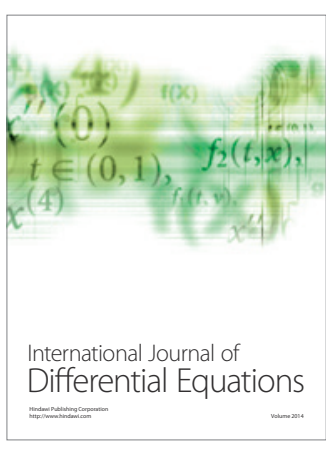
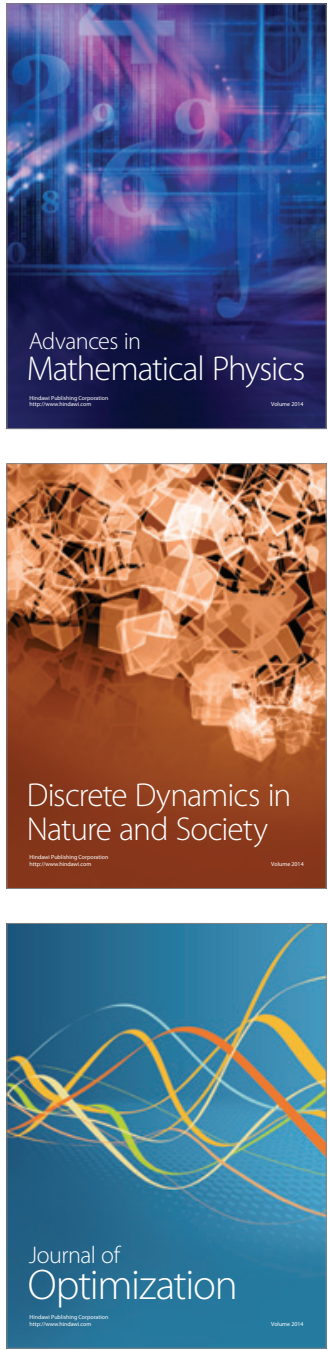